\title{
Combining multiple serum biomarkers in tumor diagnosis: A clinical assessment
}

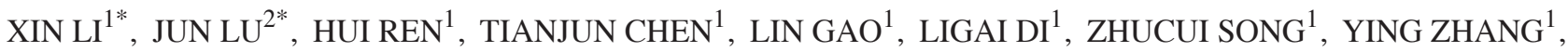 \\ TIAN YANG $^{1}$, ASMITANANDA THAKUR $^{1}$, SHU-FENG ZHOU $^{3}$, YANHAI YIN ${ }^{1}$ and MINGWEI CHEN ${ }^{1}$ \\ ${ }^{1}$ Department of Respiratory Medicine, The First Affiliated Hospital of Medical School, Xi'an Jiaotong University, \\ Xi'an, Shaanxi 710061, P.R. China; ${ }^{2}$ School of Interdisciplinary Health Studies, \\ Faculty of Health and Environmental Sciences, Auckland University of Technology, Auckland 1142, New Zealand; \\ ${ }^{3}$ College of Pharmacy, University of South Florida, Tampa, FL 33612, USA
}

Received June 20, 2012; Accepted August 20, 2012

DOI: $10.3892 / \operatorname{mco} .2012 .23$

\begin{abstract}
The present study aimed to assess the diagnostic/ prognostic value of various clinical tumor markers, including carcinoembryonic antigen (CEA), neuron-specific enolase (NSE), cytokeratin 19 (CYFRA21-1), $\alpha$-fetoprotein (AFP), carbohydrate antigen-125 (CA-125), carbohydrate antigen19.9 (CA-19.9) and ferritin, individually or in combination. The electro-chemiluminescence immunization method was performed to detect the levels of seven tumor markers in 560 cancer patients and 103 healthy subjects for comparison. The serum levels of the seven markers measured in cancer patients were higher compared to healthy subjects $(\mathrm{P}<0.05$ for AFP and $\mathrm{P}<0.001$ for the remaining six markers). Different markers had different sensitivity towards different types of tumors. Combining more markers significantly increased the ratios of positive diagnosis in the tumors. The diagnostic sensitivities of combining seven markers were particularly high in digestive, urinary and skeletal tumors (82, 92 and 83\%, respectively). Gynecological tumors have exhibited a constant yet relatively low positive diagnosis irrespective of the use of a single marker or combined markers. However, the increase in sensitivity when combining markers was accompanied by a decrease in specificity. Generally, combining more markers
\end{abstract}

Correspondence to: Professor Mingwei Chen, Department of Respiratory Medicine, The First Affiliated Hospital of Medical School, Xi'an Jiaotong University, 277 West Yanta Rd, Xi'an 710061, Shaanxi, P.R. China

E-mail: chenmingwei@mail.xjtu.edu.cn

Dr Jun Lu, School of Interdisciplinary Health Studies, Faculty of Health and Environmental Sciences, Auckland University of Technology, Private Bag 92006, Auckland 1142, New Zealand E-mail: jun.lu@aut.ac.nz

*Contributed equally

Key words: tumor marker, diagnosis, lung cancer, carcinoembryonic antigen, neuron-specific enolase, cytokeratin 19 increased the tumor detection rates, while a combination of the seven markers provided the highest detection rate. Combined detection showed a particularly high sensitivity in detecting respiratory, digestive and urinary system tumors, with the lowest sensitivity observed in gynecological tumors. As a result, combining tumor markers may play an important role in early tumor detection/diagnosis while the loss of specificity can be tolerated.

\section{Introduction}

Early diagnosis is known to lead to successful treatment of cancer (1). However, many patients are still diagnosed with late stage cancer when they seek medical care, and therefore do not receive effective treatment. Diagnosis of the disease merely by symptoms and physical signs is limiting, and the detection of tumors without any early signs is extremely difficult. Obtaining tissues for biopsy is a method employed for final diagnosis. However, this method cannot be readily repeated, as tissue biopsy causes physical harm to the patient. Moreover, unless presented with evidence of a high probability of having cancer, patients are also reluctant to undergo biopsy. Therefore, an emphasis has been placed on tumor markers and their role in assisting with screening and diagnosing tumors (2-5). Certain tumor markers are detected in serum when tumors are at an early stage, rendering the identification of tumor markers an area of interest.

A number of tumor diagnostic biomarkers have been identified and developed, a number of which have been widely adopted in the screening and diagnosis of cancer. Studies have focused on carcinoembryonic antigen (CEA) for over 40 years (6), suggesting it be a useful tool in the diagnosis of cancer (7), as it is a cancer cell adhesion marker $(8,9)$. The neuron-specific enolase (NSE) is a highly specific marker for neurons (10), with its serum level being a potentially useful marker for the detection of tumors of neuronal origin $(11,12)$. Cytokeratin 19 (CYFRA21-1) is another useful tumor marker employed in the diagnosis of various types of cancer (13-15), and is particularly sensitive in adenocarcinomas in the gastrointestinal tract and pancreas $(13,16)$. Since the 1970 's, the $\alpha$-fetoprotein (AFP) marker has been used mainly to detect gastrointestinal and 
hepatic cancer $(2,17)$. Combined with ultrasonography, AFP is the most common clinical approach to diagnosing hepatocellular carcinoma (18). Serum carbohydrate antigen-125 (CA-125) and carbohydrate antigen-19.9 (CA-19.9) are also commonly used biomarkers in the screening of various types of cancer (19-21), and are particularly sensitive to cancer in the digestive system (22-24). Ferritin is another commonly used clinical cancer biomarker, as cancer may alter the whole body iron metabolism, occasionally reflected in the change of the serum ferritin levels $(25,26)$.

The above-mentioned tumor markers exhibit various sensitivities against various types of cancer. Generally, each marker has a relatively low sensitivity, albeit a reasonable specificity towards certain types of cancer (4). Since these tumor markers can be measured using electrochemiluminescence immunization, a combination of these tumor markers is likely to improve the clinical tumor diagnostic sensitivity. In the present study, various combinations of the seven tumor markers were examined and the clinical diagnostic values of those combinations were assessed.

\section{Materials and methods}

Patient cohorts. This study was approved by the Human Ethics Committee of Xi'an Jiaotong University. In total, 560 malignant tumor cases from out- and inpatients of the First Hospital of the Xi'an Jiaotong University were gathered between June, 2008 and December, 2009. The patients were firmly diagnosed using pathohistological methods. The number of patients and tumor types are listed in Table I. None of the subjects showed severe damage of other organs, had diabetes, hypertension, or dysfunction of the heart, liver or kidney. The control group comprised 103 healthy idividuals (44 males and 59 females; median age, 55.3 years) who underwent physical examination at the hospital.

Detection method. Peripheral blood (2.0 ml) was collected in a vacuum tube (BD Biosciences, Franklin Lakes, NJ, USA) without hematolysis or lipemia from each participant after overnight fasting. Each sample was centrifuged at 1,710 x g for $5 \mathrm{~min}$ at $4^{\circ} \mathrm{C}$. The serum was separated from the sample following centrifugation, and kept at $4^{\circ} \mathrm{C}$ until analysis. The assays were performed within 5 days after samples were obtained. Electrochemiluminescence immunization (Roche Cobas e601) was applied to evaluate the seven tumor markers.

Assessment of the results. The values in the assay kits provided by the manufacturers were accepted as the clinical cut-off values. The cut-off values of the markers were as follows: CEA $<3.4 \mathrm{ng} / \mathrm{ml}$, CA- $125<35 \mathrm{U} / \mathrm{ml}$, CA-19.9 $<39$ U/ml, AFP $<7.02 \mathrm{ng} / \mathrm{ml}$, CYFRA $21-1<3.3 \mathrm{ng} / \mathrm{ml}$, $30 \mathrm{ng} / \mathrm{ml}<$ ferritin $<400 \mathrm{ng} / \mathrm{ml}$ and NSE $<15.2 \mu \mathrm{g} / \mathrm{l}$. The upper bound of the normal value was the critical value. When the value was higher than the critical value, it was considered to be positive (indicating the presence of tumor). We used ' $a$ ' to represent the true positive value; ' $b$ ', the false positive value; 'c', the false negative value and ' $d$ ', the true negative value. The sensitivity was calculated using the formula: $\mathrm{a} /(\mathrm{a}+\mathrm{c}) \times 100 \%$, while the specificity was calculated as $d /(b+d) \times 100 \%$. When combining tumor markers, a positive in any marker was considered to be overall positive.

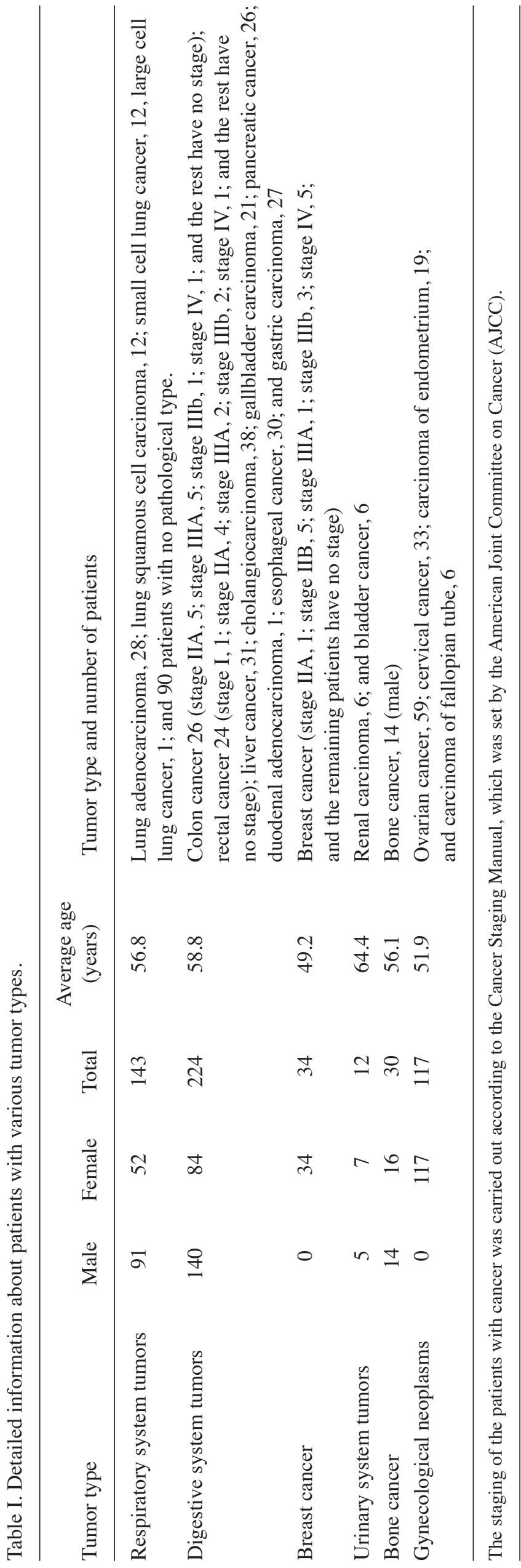


Statistical analysis. Statistical analysis was performed using the SPSS v13.0 software. $\mathrm{P}<0.05$ was considered to indicate a statistically significant difference. The median value was taken, since some of the data exceeded the standard curve end values and the tumor marker levels did not show normal distribution. The $\chi^{2}$ test was used to compare the ratio.

\section{Results}

Levels of serum tumor markers in subjects with and without cancer. The serum levels of the seven tumor markers in subjects with and without tumors are listed in Table II. The serum levels of the seven tumor markers were significantly higher compared to the healthy controls with all P-values $<0.001$, with the exception of the P-value of AFP, which was $<0.05(0.037)$.

Diagnostic efficiency in various tumors. The positive ratios of the seven tumor markers in cancer patients were significantly higher compared to the healthy subjects, with P-values $<0.001$, with the exception of AFP, which was 0.002 (Table III). The positive ratios of tumor markers in the various groups of tumors were significantly different $(\mathrm{P}<0.05)$. The detection rates of CYFRA21-1, CEA, NSE and CA-125 were relatively high in the respiratory system tumor group ( $>40 \%)$; in the digestive system tumor group (CA-19.9, CEA and CYFRA21-1) $(>40 \%)$ and in the urinary system tumor group (CA-125) $(50 \%)$. However, the number of tumor cases was relatively low (only 12), whereas CYFRA21-1 and NSE in the skeletal system tumor group had relatively high detection rates $(>40 \%)$ (Table III). The false positive rates of the seven tumor markers in different tumor groups were 1.0-11.8\% (Table III).

Combining tumor markers in diagnosis. Tumor markers were combined to increase detection sensitivity. Different combinations of tumor markers were assessed and sensitivities detected (Table IV). Generally, with an increased number of tumor markers, the detection sensitivity increased (Table IV). However, increased sensitivity also meant decreased specificity (Table IV). The highest sensitivity (97.6\%) was achieved by combining six tumor markers (CEA, NSE, CYFRA21-1, CA-125, CA-19.9 and ferritin) in respiratory system tumors. Adding the seventh marker, AFP, did not increase the sensitivity. However, the specificity was low for such a six-marker combination (65.7 or 67/102), meaning 35 out of 102 healthy individuals could be identified as potential cancer patients. In the digestive system tumors, urinary system tumors and bone cancers, the detection rates were also high when the seven tumor markers were combined $(82.2,90.9$ and $83.3 \%$, respectively). However, the specificity for such a combination was only $64.7 \%$ (Table IV).

Tumor markers in different tumors. Tumors in different systems were identified, and the detection rates of the tumor markers for those tumors are shown in Table V. CYFRA21-1 was the best predictor for lung squamous cell carcinoma, with an $83.33 \%$ positive rate. CA-19.9 was the best predictor for gallbladder carcinoma, with a $71.43 \%$ positive rate. CEA was the best predictor for pancreatic cancer, with a $61.54 \%$ positive rate. The worst prediction rates of the seven tumor markers were evident mainly in cervical cancer and carcinoma of the fallopian tube.

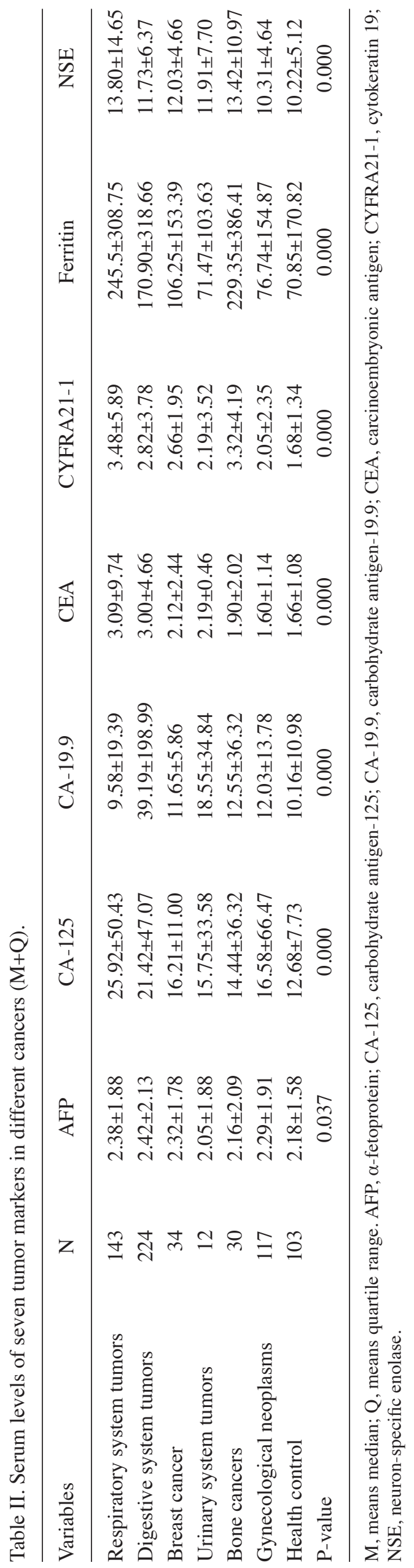




\section{Discussion}

Seven serum tumor markers were measured in 560 cancer patients and 103 healthy volunteers to study the sensitivity and specificity of these markers. Findings showed that different markers had different detection sensitivities in different groups of tumors. Combining tumor markers significantly increased the detection sensitivity, while markedly decreasing specificity.

Results of the present study have shown that, as a single marker, the CEA-positive ratio is the highest in respiratory system tumors, at $46.2 \%$ (Table III), while according to specific tumors analyzed, CEA in pancreatic cancer exhibited the highest sensitivity, at $61.5 \%$ (Table V). However, the detection sensitivity of CEA was found to be relatively low in the urinary system and gynecological tumors (8.3 and 6.4\%, respectively). The specificity of CEA in this study was shown to be relatively good, reaching $96.1 \%$ (false-positive rate $3.9 \%$ ) (Table III). Previous studies have shown that CEA levels are elevated in 0-38\% limited-stage small cell lung cancer (SCLC) patients and $40-65 \%$ in extensive-stage SCLC patients (27), as well as in $30-65 \%$ of non-SCLC patients (28). Results of another study have demonstrated that CEA levels were altered in glandular cancer with a high positive ratio and specificity (29). However, CEA has also been shown to be elevated in some benign tumors and non-tumor diseases, including hepatic cirrhosis, colonic polyp, smoking and pregnancy (30). Therefore, the specificity of CEA poses a problem. However, in this study, individuals with other diseases were excluded from our healthy volunteer group, resulting in a high specificity (Table III).

Results showed the positive ratio of CYFRA21-1 to be the highest in respiratory system tumors in this study, especially in lung squamous cell carcinoma (Table $\mathrm{V}$ ). This ratio is also high in bone cancers and tumors of the digestive system. Therefore, it may be considered as an index for the early diagnosis of lung cancer, osteocarcinoma and digestive system tumors. CYFRA21-1 is widespread in epithelial cells (31). When epithelial cells malignantly transform, an activated protease accelerates the degradation of keratin and a large amount of keratin dissolvable CYFRA21-1 fragments is released into the blood circulation, thus the serum concentration of CYFRA21-1 increases (32). CYFRA21-1 has been reported to be higher in patients with squamous cell carcinoma compared to patients with glandular and SCLC (33).

The positive ratio of CA-19.9 is the highest in digestive system tumors, followed by urinary system tumors. Specifically, the carcinoma of the gallbladder shows the highest sensitivity, while other system tumors show low sensitivity (Table V). Our data indicate that CA-19.9 may be used as a clinical index for the early diagnosis of digestive system and urinary system tumors. In the literature, there are suggestions that CA-19.9 be considered a good marker for pancreatic cancer (34), which is inconsistent with our results, where the positive rate of CA-19.9 in pancreatic cancer was relatively high, at $57.7 \%$ (Table V).

Findings of this study indicate that the positive ratio and serum level of NSE are the highest in respiratory system tumors (Tables II and III), while in the digestive system and breast tumor groups, NSE sensitivity is low. As an isozyme of enolase, NSE has been confirmed by a large amount of clinical data to be the most valuable tumor marker for SCLC, and is

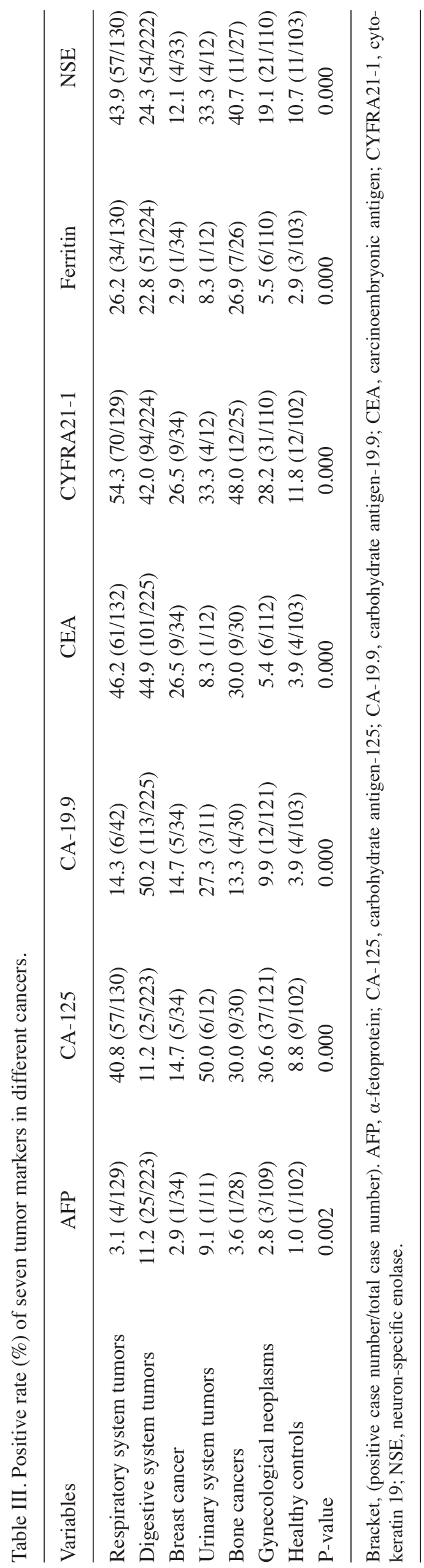




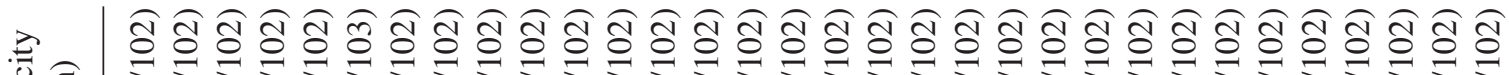

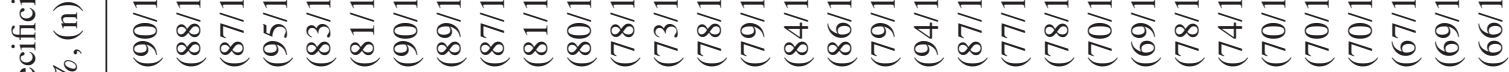
o

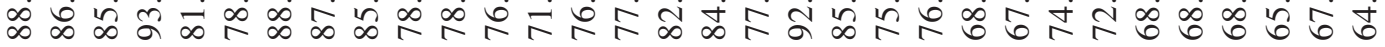

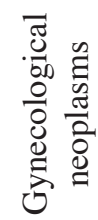

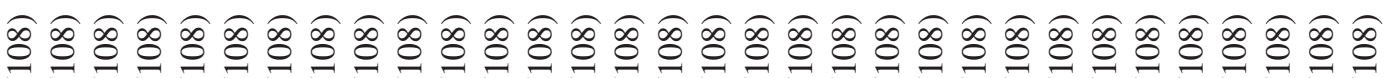

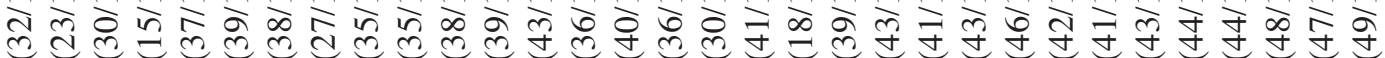

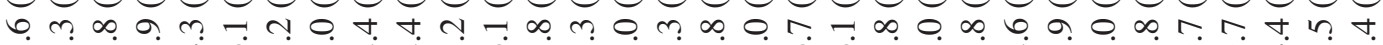

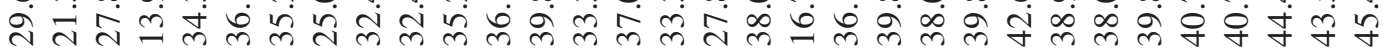

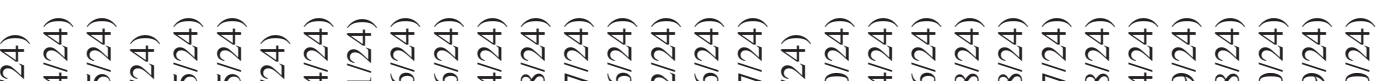

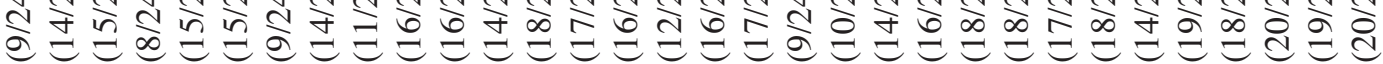
n

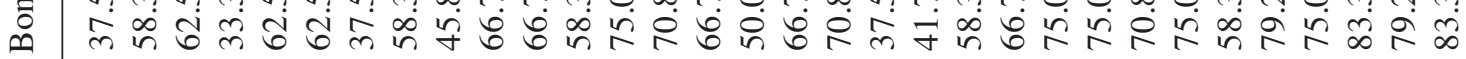
₹

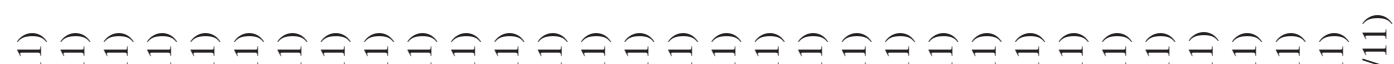

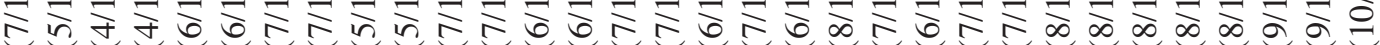

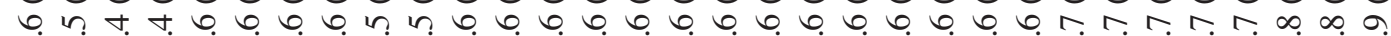
வे 守

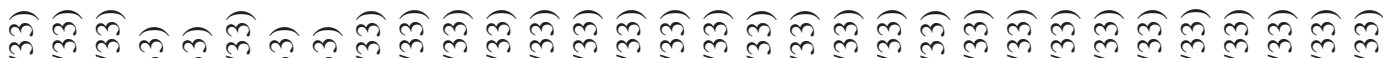
$\exists$ dे

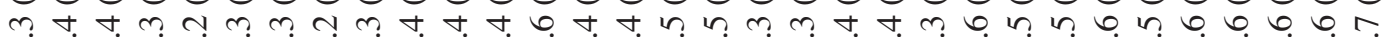

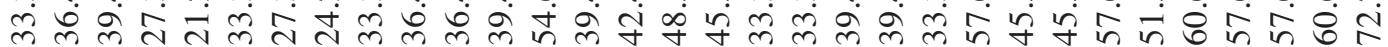
ต

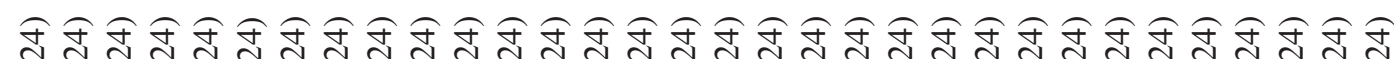

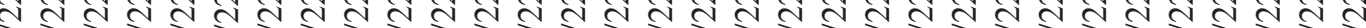
ปิ

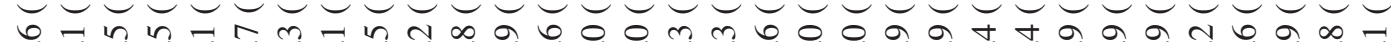

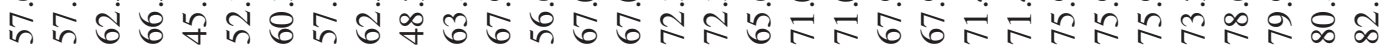

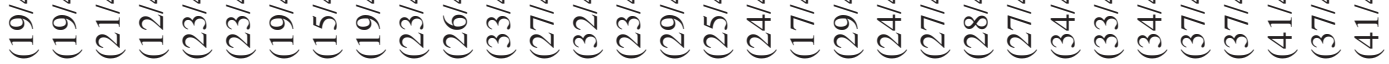

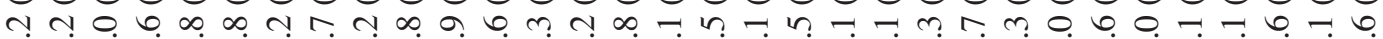

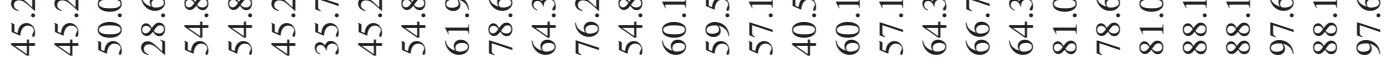




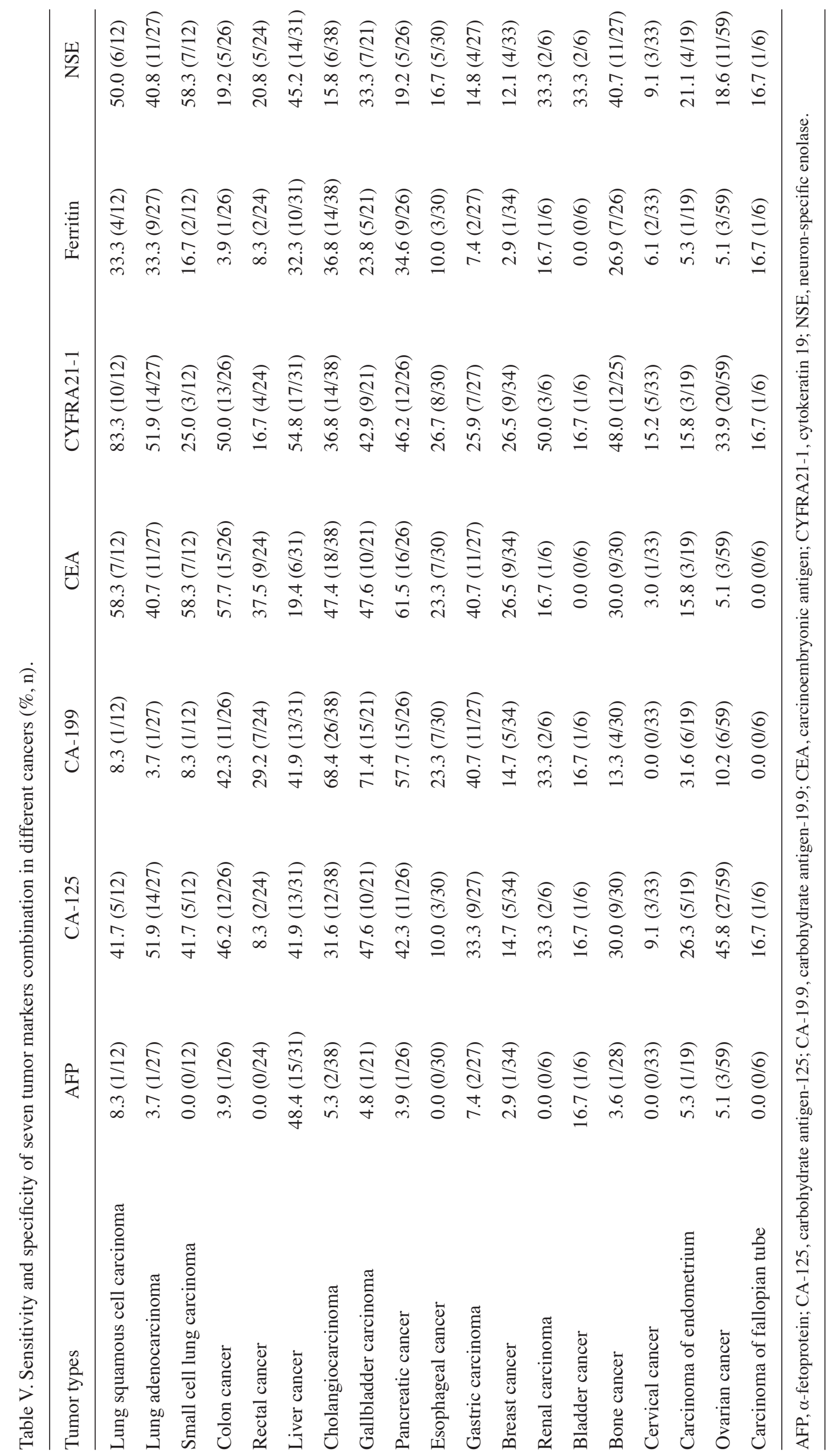


considered the most sensitive and specific marker for SCLC (35). In our study, the detection rate of NSE in SCLC is one of the highest among the seven markers and the highest compared to NSE in other types of tumors (58.3\%) (Table V). However, the specificity is low in our study, with the false-positive ratio reaching $10.3 \%$ (Table III).

Our study has shown that the positive ratios of ferritin are not high in tumors of all the systems. This may be due to the fact that ferritin is present in various tissues and inflammation may elevate its level. Therefore, it may not be valuable in diagnosing tumors alone, but should be considered as an assistant reference index. Synthesizing and enriching in the liver, a large amount of ferritin is released when liver cells are damaged or destroyed due to liver diseases or tumors. Since the response is not tumor-specific, the specificity is generally low (36). In recent years, ferritin has been found to be synthesized and secreted by several entity malignant tumors, thus it may be considered an auxiliary diagnostic criterion for malignant tumors. Early literature suggests that the serum ferritin level in patients with leukemia, Hodgkin's disease, breast cancer, ovarian cancer and colon carcinoma markedly increases and is correlated with the degree of the disease $(37,38)$. In our study, the specificity of ferritin in healthy volunteers was high with the false-positive ratio, reaching only $2.9 \%$ (Table III). This finding may be because only healthy subjects were studied and individuals with other liver diseases were excluded.

In our study, urinary system tumors have the highest CA-125-positive detection ratio, followed by the respiratory system tumors. This finding may be considered a diagnostic index for tumors of the respiratory and the urinary system. However, previous studies have raised doubts regarding the specificity of CA-125 in malignant tumors, as it is present in the mammary gland, the lung and benign and malignant effusions $(39,40)$. Another study has indicated that the sensitivity and specificity of CA-125 in diagnosing early ovarian cancer are 72 and $95 \%$, respectively (41). Apart from ovarian tumors, CA-125 has also been confirmed to have a high positive detection ratio in lung cancer patients (11). CA-125 is expressed in $\sim 51 \%$ of lung cancer tissues, and the level is closely correlated with the differentiated degree of tumors (42). In non-SCLC patients, the CA-125 level is elevated, especially in advanced-stage patients (43). In our study, the CA-125-positive detection ratio appeared to be high $(>40 \%)$ in lung squamous cell carcinoma, lung adenocarcinoma, SCLC, colon and liver cancer, gall bladder carcinoma and ovarian cancer (Table V). The positive ratio of CA-125 in ovarian cancer was found to be the highest among seven markers, suggesting that it may be used for the diagnosis of ovarian cancer. However, the specificity of CA-125 in our results was relatively low, with an $8.8 \%$ false-positive ratio in the healthy controls (Table III).

In this study, the positive ratio of AFP was the highest in digestive system tumors, especially in liver cancer. The detection ratio in liver cancer was significantly higher compared to any other type of tumor. This is inconsistent with the literature, where AFP is considered to be the specific diagnosis index for liver cancer (44). When combined with other markers, AFP sensitivity increases significantly (44). However, AFP also elevates in non-tumor diseases, such as acute and chronic hepatitis and cirrhosis (45).
In our study, CEA+CYFRA21-1 shows a higher overall sensitivity compared to any other two-marker combinations. CEA+CA-125 shows the highest sensitivity in urinary system tumors, while the sensitivity of CEA+CYFRA21-1 is the highest in breast tumors. In bone cancer, CEA+NSE shows the highest sensitivity. In the digestive system tumors, the sensitivity of CEA+CA-19.9 is the highest compared to the other two-marker combinations. Furthermore, the specificity of CEA+CA-19.9 in healthy subjects is high, rendering this combination a favorable option for the diagnosis of digestive tumors. Since the threemarker combination does not significantly increase sensitivity, the CEA+CYFRA21-1 and CEA+CA-19.9 combination may be more valuable in the clinical diagnosis of tumors in the respiratory and the digestive system. However, the present study includes only limited cases, thus additional large-scale studies are needed to explore/confirm the diagnostic potential of those markers and combinations.

A combination of more markers, leads to an increase in the detection rate. Results of the present study have shown that combining seven markers yields the highest detection ratios, i.e., 97.6, 82.1, 72.7, 90.9, 83.3 and 45.4\%, in respiratory and digestive system tumors, breast cancer, urinary system tumors, bone cancer and gynecological tumors, respectively. It appears that the seven-marker combination provides a high sensitivity in detecting tumors in the respiratory, digestive, urinary and bone systems. However, the combination of seven markers to detect tumors compromises specificity. The lowest specificity of a single marker is $88.2 \%$ (CYFRA21-1). The combination of seven markers resulted in the decrease of specificity to $64.7 \%$ (Table IV). This renders combination markers less useful in detecting breast cancer and gynecological tumors, where combined sensitivity is only 72.7 and $45.4 \%$, respectively. No statistically significant difference was detected between the ratios of false-positive and true-positive. The decrease in specificity raises doubt regarding the usefulness of the serum marker combination in tumor diagnosis, even for detecting tumors with high sensitivity. With the inpatient number of the oncology department reaching $\sim 5,000 /$ year in our hospital, the potential false-positive number would have been significant, should such seven-marker combination be used for patient diagnosis. Thus, the combination of multiple markers in diagnosis may be more suitable for patients with suspected tumors.

In conclusion, due to the complexity of the tumor origin and the heterogeneity of the tumor antigen expression, a single tumor marker may not be able to sufficiently detect tumors in certain cases. The combination of several markers may be helpful to improve diagnosis. However, a decreased specificity is an issue that needs to be addressed when employing such an approach. Considering the diversification of the biological behavior of tumor cells and the possibility of heterogenization in the course of treatment, tumor markers should be combined and detected repeatedly, in order that they more closely reflect the oncocytology and improve diagnostic efficiency.

\section{Acknowledgements}

This study was financed by the National Natural Science Foundation of China (grant no. 30973412), the Lottery Grants Board Health Research of New Zealand (J.L.) and the Faculty 
of Health and Environmental Sciences of the Auckland University of Technology (J.L.).

\section{References}

1. Welch HG, Schwartz LM and Woloshin S: Are increasing 5-year survival rates evidence of success against cancer? JAMA 283: 2975-2978, 2000.

2. Bates SE and Longo DL: Use of serum tumor markers in cancer diagnosis and management. Semin Oncol 14: 102-138, 1987.

3. Kluger HM, Hoyt K, Bacchiocchi A, et al: Plasma markers for identifying patients with metastatic melanoma. Clin Cancer Res 17: 2417-2425, 2011

4. Cohn SL, Lincoln ST and Rosen ST: Present status of serum tumor markers in diagnosis, prognosis, and evaluation of therapy. Cancer Invest 4: 305-327, 1986.

5. Virji MA, Mercer DW and Herberman RB: Tumor markers in cancer diagnosis and prognosis. CA Cancer J Clin 38: 104-126, 1988.

6. Maxwell P: Carcinoembryonic antigen: cell adhesion molecule and useful diagnostic marker. Br J Biomed Sci 56: 209-214, 1999.

7. Molina R, Filella X, Auge JM, et al: Tumor markers (CEA, CA 125, CYFRA 21-1, SCC and NSE) in patients with non-small cell lung cancer as an aid in histological diagnosis and prognosis Comparison with the main clinical and pathological prognostic factors. Tumour Biol 24: 209-218, 2003.

8. Hammarstrom S: The carcinoembryonic antigen (CEA) family: structures, suggested functions and expression in normal and malignant tissues. Semin Cancer Biol 9: 67-81, 1999.

9. Tan E, Gouvas N, Nicholls RJ, et al: Diagnostic precision of carcinoembryonic antigen in the detection of recurrence of colorectal cancer. Surg Oncol 18: 15-24, 2009.

10. Carney DN and Teeling M: Neuron-specific enolase: how usefu as a cancer marker? Eur J Cancer Clin Oncol 24: 825-828, 1988.

11. Hatzakis KD, Froudarakis ME, Bouros D, et al: Prognostic value of serum tumor markers in patients with lung cancer. Respiration 69: 25-29, 2002.

12. Cooper EH: Neuron-specific enolase. Int J Biol Markers 9: 205-210, 1994.

13. Saloustros E and Mavroudis D: Cytokeratin 19-positive circulating tumor cells in early breast cancer prognosis. Future Onco 6: 209-219, 2010.

14. Uenishi T, Kubo S, Hirohashi K, et al: Cytokeratin-19 fragments in serum (CYFRA 21-1) as a marker in primary liver cancer. Br J Cancer 88: 1894-1899, 2003.

15. Stieber P, Hasholzner U, Bodenmuller H, et al: CYFRA 21-1: a new marker in lung cancer. Cancer 72: 707-713, 1993.

16. Jain R, Fischer S, Serra S, et al: The use of cytokeratin 19 (CK19) immunohistochemistry in lesions of the pancreas, gastrointestinal tract, and liver. Appl Immunohistochem Mol Morphol 18: $9-15,2010$

17. Groover JR and Rogers AI: Immunologic tests for the detection of gastrointestinal cancers: status report on carcinoembryonic antigen (CEA) and alpha-fetoprotein (AFP). Southern Med J 66: 1218-1221, 1973.

18. Chen L, Ho DW, Lee NP, et al: Enhanced detection of early hepatocellular carcinoma by serum SELDI-TOF proteomic signature combined with alpha-fetoprotein marker. Ann Surg Oncol 17: 2518-2525, 2010.

19. Kemer T, Ormen M, Kuralay F, et al: Diagnostic usefulness of carbohydrate antigen-125 in cancerous and noncancerous peritoneal effusions. Tohoku J Exp Med 205: 11-18, 2005.

20. Oktay G, Guner G, Karlikaya C and Akkoclu A: Serum levels of neuron-specific enolase, carbohydrate antigen 19-9 and carbohydrate antigen 125 in carcinoma of the lung. Biochem Soc Trans 23: 211S, 1995 .

21. Yeh LS, Hung YC, Kao A, et al: Tissue polypeptide specific antigen (TPS) and carbohydrate antigen 125 (CA-125) in the early prediction of recurrent ovarian cancer. Anticancer Res 22: 3669-3671, 2002.

22. Encabo G, Genolla J, Gefaell R and Ruibal A: Serum carbohydrate antigen 19.9 in patients with digestive cancers. Presse Med 14: 2304, 1985 (In French).

23. Halloran CM, Ghaneh P, Connor S, et al: Carbohydrate antigen 19.9 accurately selects patients for laparoscopic assessment to determine resectability of pancreatic malignancy. Br J Surg 95: 453-459, 2008.
24. van Ruth S, Hart AA, Bonfrer JM, et al: Prognostic value of baseline and serial carcinoembryonic antigen and carbohydrate antigen 19.9 measurements in patients with pseudomyxoma peritonei treated with cytoreduction and hyperthermic intraperitoneal chemotherapy. Ann Surg Oncol 9: 961-967, 2002.

25. Torti SV and Torti FM: Iron and ferritin in inflammation and cancer. Adv Inorg Biochem 10: 119-137, 1994.

26. Richardson DR: Iron and neoplasia: serum transferrin receptor and ferritin in prostate cancer. J Lab Clin Med 144: 173-175, 2004.

27. Slodkowska J, Szturmowicz M, Rudzinski P, et al: Expression of CEA and trophoblastic cell markers by lung carcinoma in association with histological characteristics and serum marker levels. Eur J Cancer Prev 7: 51-60, 1998.

28. Bergman B, Brezicka FT, Engstrom CP and Larsson S: Clinical usefulness of serum assays of neuron-specific enolase, carcinoembryonic antigen and CA-50 antigen in the diagnosis of lung cancer. Eur J Cancer 29A: 198-202, 1993.

29. Seemann MD, Seemann O, Dienemann H, Schalhorn A, Prime G and Fink U: Diagnostic value of chest radiography, computed tomography and tumour markers in the differentiation of malignant from benign solitary pulmonary lesions. Eur J Med Res 4: 313-327, 1999.

30. Schneider J and Schulze G: Comparison of tumor M2-pyruvate kinase (tumor M2-PK), carcinoembryonic antigen (CEA), carbohydrate antigens CA 19-9 and CA 72-4 in the diagnosis of gastrointestinal cancer. Anticancer Res 23: 5089-5093, 2003.

31. Buccheri G, Torchio P and Ferrigno D: Clinical equivalence of two cytokeratin markers in non-small cell lung cancer: a study of tissue polypeptide antigen and cytokeratin 19 fragments. Chest 124: 622-632, 2003.

32. Sung HJ and Cho JY: Biomarkers for the lung cancer diagnosis and their advances in proteomics. BMB Rep 41: 615-625, 2008.

33. Muraki M, Tohda Y, Iwanaga T, Uejima H, Nagasaka Y and Nakajima S: Assessment of serum CYFRA 21-1 in lung cancer. Cancer 77: 1274-1277, 1996.

34. Gaspar MJ, Arribas I, Coca MC and Diez-Alonso M: Prognostic value of carcinoembryonic antigen, CA 19-9 and CA 72-4 in gastric carcinoma. Tumour Biol 22: 318-322, 2001.

35. Pujol JL, Boher JM, Grenier J, Quantin X, Saffont L and Daures JP: Neuron-specific enolase (NSE) in the surveillance of small cell cancers: an evaluation of the prognostic information using Markov's model. Rev Mal Respir 15: 519-525, 1998 (In French).

36. Chou SF and Chen CY: Monoclonal and polyclonal antibodies against human ferritin, a nonspecific tumor marker. Hybridoma 20: 59-62, 2001.

37. Aulbert E and Schmidt CG: Ferritin - a tumor marker in myeloid leukemia. Cancer Detect Prev 8: 297-302, 1985.

38. Dorner MH, Abel U, Fritze D, Manke HG and Drings P: Serum ferritin in relation to the course of Hodgkin's disease. Cancer 52: 2308-2312, 1983

39. Bekci TT, Senol T and Maden E: The efficacy of serum carcinoembryonic antigen (CEA), cancer antigen 125 (CA125), carbohydrate antigen 19-9 (CA19-9), carbohydrate antigen 15-3 (CA15-3), alpha-fetoprotein (AFP) and human chorionic gonadotropin (hCG) levels in determining the malignancy of solitary pulmonary nodules. J Int Med Res 37: 438-445, 2009.

40. Wu GP, Ba J, Zhao YJ and Wang EH: Diagnostic value of CEA, CYFRA 21-1, NSE and CA 125 assay in serum and pleural effusion of patients with lung cancer. Acta Cytol 51: 679-680, 2007.

41. Donach M, Yu Y, Artioli G, et al: Combined use of biomarkers for detection of ovarian cancer in high-risk women. Tumour Biol 31: 209-215, 2010.

42. Goldenberg DM, Sharkey RM and Primus FJ: Immunocytochemical detection of carcinoembryonic antigen in conventional histopathology specimens. Cancer 42: 1546-1553, 1978.

43. Nunez GR, Ito C and Del Giglio A: Increased serum CA-125 levels in patients with lung cancer post thoracotomy. Southern Med J 102: 427-428, 2009.

44. Beneduce L, Castaldi F, Marino M, et al: Improvement of liver cancer detection with simultaneous assessment of circulating levels of free alpha-fetoprotein (AFP) and AFP-IgM complexes. Int J Biol Markers 19: 155-159, 2004.

45. Beale G, Chattopadhyay D, Gray J, et al: AFP, PIVKAII, GP3, SCCA-1 and follisatin as surveillance biomarkers for hepatocellular cancer in non-alcoholic and alcoholic fatty liver disease. BMC Cancer 8: 200, 2008. 